\title{
La experiencia del humor en personas con discapacidad intelectual
}

\section{Daniel Paredes Gómez}

Departamento de Psicología. Centro Ocupacional Aprosuba-4 (FEAPS)

\section{España}

dapago@cop.es

Dr Daniel Paredes Gómez. Departamento de Psicología. Centro Ocupacional Aprosuba-4 (FEAPS). C/ Don Llorente, 31, Don Benito, Badajoz. España. E-mail: dapago@cop.es

(C) Education \& Psychology I+D+i and Editorial EOS (Spain) 


\section{Resumen}

El presente trabajo se centra en el estudio del sentido del humor de las personas con discapacidad intelectual. El tema se introduce con una aproximación a algunas de las principales teorías, definiciones y modelos con los que se ha intentado explicar psicológicamente este fenómeno humano; para luego ir vinculando estos conocimientos, desde una perspectiva evolutiva, a la discapacidad intelectual. Se revisan los resultados de varias investigaciones llevadas a cabo sobre la apreciación del sentido del humor en las que se comparan grupos, la mayoría infantiles, con diversas características como el autismo, los problemas de aprendizaje y la discapacidad intelectual. A continuación el tema se centra más detalladamente en las personas adultas con discapacidad intelectual, analizando algunos condicionantes que concurren en su apreciación y en su comportamiento humorístico. Y, por último, se establecen varias conclusiones entre las que destacan que el sentido del humor, también, es un asunto muy importante para el bienestar emocional de las personas con discapacidad intelectual y la necesidad de ampliar los conocimientos que se tienen actualmente sobre este tema.

Palabras Clave: sentido del humor, discapacidad intelectual, emociones positivas. 


\begin{abstract}
The article focuses on the study of the positive emotion of humor in persons with intellectual disability. The subject is introduced a brief look at some of the main theories, definitions and models used in attempting to explain psychologically this human phenomenon; in order to link them to the general characteristics of intellectual disability, from an evolutionary perspective. Results from several studies on sense of humor are reviewed, where groups are compared, largely groups of children, with different characteristics such as autism, learning disability and intellectual disability. Next, more detailed attention is given to adult persons with intellectual disability, analyzing some of the conditioning factors that influence their appreciation of humor and their own humor-related behavior. Finally, several conclusions are drawn, including that sense of humor is a very important matter for the emotional well-being also of the intellectually disabled, and the need to expand our current knowledge of this topic.
\end{abstract}

Keywords: sense of humor, intellectual disability, positive emotions.

Manuscript received: 04/27/07 Initial Acceptance: 08/21/07 Final Acceptance: 09/28/07 


\section{Introducción}

Los esfuerzos por entender el fenómeno humano del sentido del humor, su apreciación y expresión, han sido reflejados en varias teorías psicológicas. Las principales se han apoyado en alguna de las siguientes ideas-fuerza: a) alivio-descarga; b) superioridad-denigración; y c) incongruencia (Carretero, Pérez y Buela, 2006; Polimeni y Reiss, 2006).

Aquellas teorías que basan sus argumentos en la primera idea, avaladas mayormente por las escuelas psicoanalíticas, entienden el sentido del humor y la risa como una expresión liberadora de los impulsos inconscientes reprimidos, que generarían tensión psicológica; el individuo precisaría, por tanto, de esta válvula de escape para no generar un caos personal.

Por su parte, las basadas en la superioridad y la denigración razonan el sentido del humor como un potente mecanismo reforzador de la autovaloración personal ante los errores, los defectos o las carencias ajenas, e incluso propias. Lo divertido se provocaría por un sentimiento de vanidad súbita que genera regocijo y risa, intentando aumentar con ello el propio estatus personal.

Las teorías basadas en la tercera idea, la incongruencia, basan su foco de interés en los aspectos cognitivos. Y resuelven que el sentido del humor se produce por la asociación mental inesperada de dos ideas o situaciones que lógicamente no deberían permanecer unidas. De manera que teniendo una estructura compleja, habría un elemento normal y otro que quebrantaría el orden subjetivo establecido; más un tercer elemento contextual que sería la sorpresa .

Estas últimas teorías están inmersas en un interesante debate referente a si la incongruencia, por si sola es una condición suficiente y necesaria para la experiencia emocional del sentido del humor a partir de su mera detección perceptiva; este sería un humor incongruente pero sin contenido, como ocurre con las payasadas. $\mathrm{O}$ si se requiere del funcionamiento de procesos cognitivos del tipo incongruencia-resolución; desarrollados en dos etapas: una la percepción de la incongruencia y otra, posterior, la reevaluación de la situación para resolverla cognitivamente de manera adecuada (Carretero et al., 2006; Glass, 2003). En cualquier caso, desde este punto de vista cognitivo, el sentido del humor implicaría una distorsión de la función, de la norma, y esa incongruencia es la que llama la atención, asombra y despierta la risa (Carbelo, 2005). 
A lo dicho habría que añadir que la expresión y/o la apreciación de lo humorístico se ven sometidas también a restricciones cualitativas en relación al contenido o la forma de presentación de los estímulos graciosos; dependiendo, incluso, del momento de presentación y del conocimiento previo del estímulo. Pues, por ejemplo, si los elementos humorísticos suponen un tenue desafió cognitivo y son muy fáciles de comprender no provocan la diversión; y, por el contrario, si son muy complejos tampoco, por ser algo excesivamente abstracto. Así mismo, si el estímulo es muy conocido su fuerza es mucho menor. O si la oportunidad en la que ocurre no encaja con el momento oportuno su efecto humorístico se reduce.

El estudio del sentido del humor en las personas con discapacidad intelectual se ha basado fundamentalmente en las dos últimas ideas-fuerza; por lo que el texto se referenciará principalmente en ellas. Habiéndose generado, aun con todo, muy poca literatura sobre el tema.

\section{La presencia del sentido del humor en los seres humanos}

El sentido del humor ha sido descrito como un fenómeno que atañe a la capacidad humana para experimentar y/o estimular unas reacciones muy específicas, la sonrisa y la risa (observable o no), y de esta manera conseguir o mantener un estado de ánimo positivo (Carbelo, 2006). Algo, además, que es considerado como una emoción; que puede utilizarse como sinónimo de una sensación de alegría; y que tiene características que le convierten en un mecanismo eficaz para afrontar situaciones y experiencias de vida (Buxman, 2004).

Desde la psicología evolucionista se han dado algunas explicaciones a la presencia del sentido del humor en los humanos. Algunos autores consideran que quizás sea la función cognitiva más compleja del reino animal y achacan haber tenido un papel intensamente relevante en el desarrollo filogenético de las habilidades y las relaciones sociales o del lenguaje de las personas (Polimeni y Reiss, 2006). De manera que gracias al sentido del humor, y a su evolución en la especie, los humanos de hoy en día podemos practicar conductas lúdicas; mantener en buen nivel la autoestima; utilizarlo como vehículo para demostrar aprecio y camaradería; ayudarse de él para el desarrollo del ingenio y la creatividad; centrar el sentido común; distender los asuntos graves de la vida; conseguir diversión y contento; ayudar a lidiar con la realidad, a veces positiva y otras negativa; y, llegado el caso, puede tener, incluso, una 
función psicoterapéutica para el individuo (Fernández, 2002; Salameh, 2004; Torradabella, y Corrales, 2002).

El sentido del humor parece ser una capacidad única de los seres humanos. Valorado en muchas, si no todas las culturas, posee una alta estima y un gran prestigio social (Carbelo, 2006); y es una de las fortalezas de la especie (Seligman, 2003). Pero a la par es un fenómeno psicológico muy complejo, pues abarca en su estructura general varias dimensiones interdependientes.

Uno de los modelos que mejor explican el constructo sentido del humor, por lo integrador y coherente que se ha demostrado tras años de sometimiento a pruebas empíricas, es el presentado por B. García (2006) y su grupo de investigación. Dicho modelo se compone de cuatro dimensiones: una, creación o generación de humor, abarca la faceta más externa y evidente del sentido del humor, y refiere directamente al conjunto de estrategias y habilidades propias para percibir la realidad de forma insólita y comunicarla de modo que provoque risa o sonrisas en los demás. Otra, apreciación del humor y disfrute de la vida, vendría a ser el tomarse la vida en serio pero con toques de humor, pensando en positivo, apreciando el sentido del humor de los demás y riéndose de uno mismo o de sus situaciones. Una tercera, afrontamiento optimista de problemas, es el uso del sentido del humor para hacer frente a los avatares, las dificultades, los contratiempos y los fracasos de la vida. Y, por último, la dimensión que hace referencia al establecimiento de relaciones positivas supone la utilización del humor como una herramienta para comunicarse y relacionarse socialmente de manera más eficaz.

Este modelo multidimensional parte del postulado de la existencia de una relación directa entre lo que se piensa, lo que se siente y cómo se comportan las personas. Se podría decir, por tanto, que el sentido del humor está provocado por el pensamiento racional como aspecto que provoca un estilo explicativo optimista y una forma de interpretar los sucesos que permite la apreciación de nuevas perspectivas. También por un aspecto emocional placentero que proporciona el sentimiento de esperanza de que la vida merece la pena vivirla. Por un aspecto conductual, que sería la forma de manifestar el propio sentido del humor (García, 2006). Y, por último, por una aspecto social, dado que el sentido del humor se activa y se multiplica en situaciones sociales determinadas (Carbelo, 2005). 
Que las personas con discapacidad intelectual se ríen y se divierten ante la presencia de determinados estímulos contextuales es fácilmente constatable. Pero, a la par, los estímulos, los comportamientos o incluso las situaciones sociales que, en general, se consideran comúnmente humorísticas para la mayoría de la población, por ejemplo contar chistes populares o reírse durante la representación de comedias teatrales, parecen ser poco frecuentes en la mayoría de ellas.

Ante esta observación, si se quiere imprecisa, cabría preguntarse ¿es su sentido del humor una experiencia distinta a la del resto de la población, o simplemente es la misma, salvando que se presentan en ella limitaciones en su apreciación, en su expresión, en su comprensión, en su utilización.... dada la discapacidad misma, tal y como ocurre en otros aspectos y dimensiones personales de estos individuos?.

Alguna claridad sobre la pregunta la arroja el hecho de que ninguno de los componentes de las definiciones y las dimensiones expuestas más arriba parecen excluir, a priori, a las personas con discapacidad intelectual. Así mismo, es irrebatible que son personas y, por ende, tampoco estarían fuera de las repercusiones que la evolución filogenética del sentido del humor ha tenido en la especie. Pero aun con todo las lagunas en el conocimiento del tema no dejan de ser abundantes y los límites sobre el mismo imprecisos.

\section{El sentido del humor desde una perspectiva evolutiva individualizada}

A la hora de abordar el estudio del sentido del humor en las personas con discapacidad intelectual es preciso tener en cuenta el momento evolutivo en el que se encuentra el individuo concreto.

Es sabido que las personas de este colectivo pasan a través de las mismas etapas de desarrollo cognitivo y emocional que el resto de la población, salvo que lo hacen con un progreso más lento e irregular, el cual la mayoría de las veces queda inconcluso (Zilger, Hodapp y Burack, 1990; en Charlot, 2005). Esto es así porque la capacidad para desarrollar adaptaciones adecuadas en cada estructura del desarrollo ontogenético dependen de las condiciones bio-psico-sociales (Dosen, 2005) y de las condiciones del entorno físico en las que se encuentre el sujeto. Las personas con discapacidad intelectual pueden verse impactadas fuertemente por determinadas variables en algunas de estas condiciones (o en todas), de tal forma que se modifique o dificulte su desarrollo psicológico. 
Autores como Rojhan et al. (1995) (en Arthur, 2003) hablan de la hipótesis emociónespecificidad, postulando que siendo propio de la naturaleza de las personas con discapacidad intelectual tener dificultades y limitaciones cognitivas también su capacidad para entender y expresar las emociones propias y comprender las ajenas estaría limitada en alguna medida.

Algunas de las más sólidas líneas de investigación sobre el sentido del humor que se han llevado a cabo desde la psicología evolutiva han propuesto determinados estadios evolutivos para este fenómeno humano; si bien, los autores advierten que sólo son patrones-guía generales pues se reconoce, por una parte, que hay mucha variabilidad de unos niños a otros y, por otra, también en cuanto a la secuenciación intrapersonal de cada estadio con respecto a la edad cronológica (Mc Ghee, 2002).

I.W. Brown (2007), siguiendo los patrones del desarrollo cognitivo, describió algunas características típicas de cada momento evolutivo del sentido del humor. Así, los bebés encuentran muy divertido el hallazgo de una nueva sensación corporal, un acto gestual repetido o el rápido movimiento espacial de un objeto ante su vista. Los niños preescolares hayan divertido la desviación y la exageración de la realidad de los objetos o las palabras. Sus conceptos mentales no están firmemente fijados aun, cualquier deformación o exageración sigue siendo posible, aunque saben bien que realmente no lo es. Los niños escolares de primaria encuentran el humor en la construcción de nuevas cosas u objetos, nuevas frases... y buscan para ellas un nuevo sentido, no habitual e incluso fantástico. Por primera vez, se comienza a percibir el humor con una lógica del pensamiento, aun muy inmadura y utilizando el simbolismo.

Los niños escolares de secundaria comienzan a apreciar el humor fonético, por ejemplo, al sustituir una letra para modificar una palabra y cambiarla de sentido, y el humor léxico con las palabras o frases de doble significado. Los adolescentes comienzan a aumentar la sofisticación lingüística del humor, exagerando y transformando el lenguaje, los objetos o la situación social para generar dobles significados y contrasentidos. Su humor, además, tiene un fuerte cariz experimental. Para los adultos, por último, lo gracioso se basa en la práctica intelectual de la lógica, en la experiencia vivida, en sus valores y actitudes, en intereses propios ya asentados, en las gentes que conocen y, en general, en ideas maduradas sobre el mundo, elaboradas desde la propia y subjetiva percepción. 
Así pues, el sentido del humor en las personas con discapacidad intelectual se vería afectado por el condicionante psicoevolutivo. El momento ontogenético, singular, intraindividual y único, en el que se encuentre cada una de las personas, marcaría una clave determinante en sus experiencias humorísticas.

Un estudio realizado con una pequeña muestra de 12 adolescentes con discapacidad intelectual moderada parece aportar alguna evidencia inicial sobre la relación de todos los aspectos ontogenéticos que se vienen mencionado. Sus autores encontraron relaciones significativas entre la edad mental y la predicción de la respuesta de risa para determinadas situaciones provocadas por su profesor (Berry, Parson, Hyde y Hilsdon, 1981).

\section{Discapacidad intelectual, apreciación del sentido del humor y desarrollo intelectual y social}

El sentido del humor puede ser clasificado tomando como base los componentes donde se soporta su expresión. De manera que puede hablarse de humor verbal, gráfico, sonoro, comportamental, etc.

En cualquier caso, la apreciación de cualquiera de estas formas de humor requiere la puesta en función de una serie de habilidades de tipo cognitivo y lingüístico; como, por ejemplo, integrar los estímulos relevantes de tipo auditivo y visual; observar el todo, dándose cuenta, a la vez, de las partes de la situación; fijar selectivamente la atención en la configuración social; secuenciar las tareas perceptivas; identificar, reconocer y resolver cognitivamente las incongruencias; utilizar habilidades simbólicas, etc. (Pickering, Pickering y Buchanan, 1987; Short, Basili y Schatschneider, 1993).

De forma que si efectivamente el sentido del humor se relaciona con los procesos intelectuales orientados a percibir, comprender y dotar de un significado psicológico a los sucesos incongruentes; y estando estas competencias limitadas en las personas que muestran discapacidad intelectual puede suponerse que tales restricciones influirán en la apreciación que hagan de los estímulos humorísticos.

Determinados trabajos de investigación parecen apoyar estas ideas. Así por ejemplo, estudios en los que se utilizaron pequeñas muestras de grupos de niños de clases escolares de 
primaria y secundaria ( $\mathrm{N}=54$, rango de edad: 7-16 años), unos sin discapacidad intelectual, otros con dificultades de aprendizaje y otros con discapacidad intelectual ligera, y que centraron sus objetivos en el sentido del humor cognitivo y verbal relacionado con la incongruencia que presentaban las adivinanzas graciosas; se encontraron evidencias de que en cualquiera de los grupos, sea cual fuere la edad, los niños con discapacidad intelectual tuvieron más baja tasa de comprensión del humor que aquellos que no tenían discapacidad (Bruno, Johnson y Simon, 1988).

Dificultades en la integración global de los componentes visuales (dibujos, viñetas....) y lingüísticos del humor también fueron encontradas en uno de los escasísimos estudios llevados a cabo con adultos jóvenes ( $\mathrm{N}=15$, rango de edad: 20-27 años) que tenían discapacidad intelectual ligera, señalando que posiblemente sea el humor expresado en soporte visual aquel que les resulte más fácil de apreciar (Brown, 1994).

A menudo, un completo aprovechamiento de la experiencia positiva del sentido del humor requiere la puesta en práctica de habilidades socio-emocionales en conjunto con las cognitivas. Algunas de tales habilidades se refieren a poder hacer juicios sobre las características de la gente, saber discriminar las posibles y distintas situaciones contextuales o tener habilidades para compartir el tiempo adecuadamente con otros (Short et al., 1993). Las personas con discapacidad intelectual tienen dificultades en el desarrollo de las conductas de adaptación social; también este aspecto puede tener influencias decisivas en la experiencia humorística.

En un estudio comparativo entre niños escolares ( $\mathrm{N}=47$, rango de edad: 8-10 años), sin y con discapacidad intelectual límite y ligera, y para el que se utilizaron viñetas gráficas en las que se podían observar situaciones humorísticas en distinto grado y otras neutras, se encontró que la mayoría de los niños con discapacidad intelectual presentaron, en general, una menor sensibilidad que sus iguales sin desventaja intelectual en la discriminación entre las viñetas humorísticas y las neutras; concluyendo las autoras que tales diferencias no solo se justificarían en razón de las limitaciones cognitivas; sino, incluso, en limitaciones relacionadas con la resolución de problemas concernientes a situaciones sociales (Short et al., 1993).

Algunos otros trabajos han incidido en la participación del componente cognitivo vinculado al social en las experiencias del sentido del humor de las personas con discapacidad 
intelectual. El autismo ha sido considerado como un trastorno que perturba las relaciones interpersonales y la comunicación, y que genera rigidez en el pensamiento y el comportamiento (Amodia de la Riva y Andrés, 2006). Suele creerse que las personas que padecen déficits relacionados con el espectro autista, incluso aquellos individuos que tienen un alto funcionamiento intelectual, no aprecian los estímulos humorísticos; pero investigaciones recientes han mostrado que poseen ciertas habilidades para apreciar algunas formas de humor verbal y no verbal (Lyons y Fitzgerald, 2004). Si bien el número de sus experiencias humorísticas parecen, por otra parte, ser menores con respecto a las personas con discapacidad intelectual sin autismo; y, por ende, deben suponerse menores, también, a la de la población ordinaria.

En efecto, algunos investigadores han trabajado con pequeñas muestras en las que compararon las interacciones naturales humorísticas y de risa en niños con autismo y niños con síndrome de Down. En un estudio observacional $(\mathrm{N}=12$, rango de edad: 3-6 años) se comparó la presencia espontánea e inducida del sentido del humor entre niños con autismo ( $\mathrm{N}=6$, de los cuales sólo dos tenían un CI de 75 puntos o menor) y niños con discapacidad intelectual por síndrome de Down. Las autoras encontraron una mayor y significativa ocurrencia de eventos humorísticos en los segundos que en los primeros (St. James y TagerFlusberg, 1994). En otro estudio posterior ( $N=35$, rango de edad: 3-6 años) de parecido diseño se encontraron resultados similares (Reddy, Williams y Vaughan, 2002). En cualquier caso ambos trabajos también coincidieron en encontrar que el humor y la risa estuvieron presentes de alguna manera en la vida diaria de todos los infantes que compusieron las pequeñas muestras utilizadas.

\section{Sentido del humor y personas adultas con discapacidad intelectual}

Los estudios sobre el sentido del humor en las personas adultas con discapacidad intelectual son muy escasos. Las bases de datos documentales arrojan gran cantidad de resultados para el tópico "sentido del humor" pero cuando éste se vincula a "discapacidad intelectual" apenas si aparecen reseñas, y la mayoría están referidas a la edad infantil. Muchas de ellas han sido expuestas más arriba. En definitiva, el estudio del sentido del humor en las personas con discapacidad intelectual es una campo muy poco desarrollado, más aun aquel que se concreta en las personas adultas. Los conocimientos disponibles actualmente parecen ser muy escasos $\mathrm{y}$, por ende, basados en pocas evidencias lo que les hace estar cargados de un amplio grado de vulnerabilidad. 
La apreciación del humor en las personas con discapacidad intelectual moderada desde la perspectiva evolutiva.

I.W. Brown (2007) propone que las habilidades en la apreciación del sentido del humor en personas adultas con discapacidad intelectual moderada suelen encuadrarse en un abanico de características que se engloban entre las propias de las últimas fases de la edad preescolar y las primeras fases propias de la adolescencia, llegando algunas personas incluso al aprecio del humor fonológico y léxico. Pero por regla general, lo que les hace gracia se debe a la utilización de la misma estructura cognitiva que usan los niños escolares de entre 5 y 7 años de edad. Sin embargo, su sentido del humor no puede ser considerado equivalente; pues pese a compartir el mismo cuerpo estructural lógico, estas personas no encuentran graciosas las mismas cosas, ni les divierte la misma temática que a los niños de estas edades. Entre otras cuestiones por que han vivido muchos más años y están bastante más familiarizados tanto con el ambiente social como con la efectividad de ejecución de las habilidades propias y ajenas.

Se ha constatado en diversas observaciones no sistemáticas realizadas en centros de apoyo (Paredes, 2005) que es frecuente verlos reír y divertirse cuando se producen alteraciones en los comportamientos previstos, dada la torpeza puntual del actor de la escena. Sus risas demostrarían, fenomenológicamente al menos, la presencia de experiencias divertidas basadas en la mera detección perceptiva del humor incongruente sin contenido alguno. $\mathrm{O}$ alegrarse con las variaciones incongruentes de las pequeñas rutinas diarias. Por ejemplo, suelen apreciar el humor cuando su psicólogo al tomar su nombre para rellenar algún formulario, mientras lo anota, pronuncia en voz alta otro nombre propio no correspondiente con el suyo.

Y también, se divierten con el uso de su imaginación y su fantasía. Por ejemplo, viendo una colección de fotografías referidas a actividades de ocio realizadas fuera del centro. Ambos de estos ejemplos apuntarían a suponer que también ellos aplican de alguna manera el mecanismo cognitivo basado en procesos del tipo incongruencia-resolución.

El comportamiento humorístico: humor positivo versus humor agresivo en las personas adultas con discapacidad intelectual.

Parecen existir evidencias de que los comportamientos del sentido del humor tienen una estructura afectiva bidimensional, encontrándose por un lado una valencia positiva, relacionada con la diversión, y otra negativa, relacionada con el rechazo (Carretero, 2006). 
Martín, Puhlik-Doris, Larsen, Gray y Weir (2003) (véase Brizzio, Carreras y Casullo, 2006) encontraron cuatro dimensiones principales de la expresión del sentido del humor en relación a su intencionalidad hacia los demás. Dos que consideraron saludables o adaptativas: sentido del humor afiliativo y de mejoramiento personal. Y otras dos entendidas como relativamente poco saludables o desadaptativas: sentido del humor agresivo y de descalificación personal.

En cualquier caso, y siguiendo a B. Carbelo (2005), el sentido del humor, cuando es socialmente positivo-adaptativo, puede ser una herramienta útil para la búsqueda de un mundo más humano y divertido. Como puede ser, también, un instrumento feroz capaz de provocar la humillación del otro, si su forma y estilo es negativo/agresivo.

Algunos registros no sistemáticos de observaciones realizadas en entornos de centros específicos de apoyo profesional muestran que en las relaciones sociales de las personas adultas con discapacidad intelectual parece ser mucho más frecuente el comportamiento humorístico positivo que el negativo (Paredes, 2005). Es decir, aquel que es provocado por el escándalo que produce todo lo que se presenta anómalo, irregular, desmesurado o torpe; dando lugar a lo ridículo pero impregnado de un halo benevolente alejado de la agresividad y la burla (Carbelo, 2005). Es decir, el que surge cuando la única intención de lo cómico es la diversión (Rodríguez, 2002).

Llamar a otra persona por el nombre del presidente del gobierno de la nación o provocar al otro mencionando que tiene una profesión poco frecuente basándose en alguna prenda del vestuario que lleva ese día o en algún detalle personal singular, pueden ser ejemplos de lo dicho.

Podría darse como primera explicación a las observaciones mencionadas que el ejercicio intelectual y comportamental-social del humor agresivo es más difícil de emitir, e incluso de apreciar, por los miembros de un colectivo socialmente débil y vulnerable, dadas sus evidentes limitaciones intelectuales y en el ejercicio práctico de su conducta adaptativa. Pero éstas y otras ideas concurrentes deben ser contrastadas empíricamente pues son sólo apuntes de observaciones tomadas del natural. 


\section{Conclusión}

Los resultados de las investigaciones y de las observaciones expuestas, aun siendo exiguos, evidencian que el sentido del humor es una experiencia positiva presente en la vida emocional de las personas con discapacidad intelectual. Y aunque es preciso reconocer que dicha experiencia está sujeta a la influencia de variables que impactan cuantitativamente tanto en el colectivo en general como de forma singular en cada persona, y que hacen referencia, por ejemplo, a las dificultades cognitivas para la apreciación de los estímulos humorísticos; no parece haber evidencias de que en sí misma la experiencia emocional sea cualitativamente menor en las personas que componen este grupo humano.

Determinados autores se han centrado en trabajar en la aplicación del sentido del humor para mejorar la calidad de vida de las personas a las que apoyan desde la psicología aplicada. Por ejemplo, se ha trabajado en el soporte positivo que supone el humor en la práctica clínica (Alemany y Cabestrero, 2002); y más específicamente en la terapia de niños y adolescentes (Bernet, 2004). También se ha aplicado el humor a la orientación vocacional y profesional (Nevo, 2004). Se ha tomado como indicador de una buena salud mental en adolescentes y adultos jóvenes (Brizzio et al, 2006). Se ha relacionado con un buen estado de salud física (Martin, 2004). O se ha utilizado en el trabajo de apoyo a familias de personas con discapacidad intelectual (Rieger, 2004).

En general, los resultados de todas estas prácticas parecen ser positivos. Aunque, a la par, se reconoce claramente que apoyarse en el sentido del humor en la práctica de la psicología también tiene sus afiladas aristas, sus muchas contraindicaciones y sus muchos riesgos profesionales (Alemany y Cabestrero, 2002).

La aplicación del sentido del humor como estrategia intencionada para mejorar el bienestar emocional de las personas con discapacidad intelectual apenas si se ha intentado; no obstante en el horizonte aparecen algunos signos alentadores. Así, en un estudio exploratorio ( $\mathrm{N}=22$, rango de edad 18-24 años) se encontró que cuando el humor era aplicado en sesiones individuales de consejo psicológico vocacional a clientes jóvenes con discapacidad intelectual moderada, y esta tarea se acompañaba de sentido del humor comprensible, apreciado espontáneamente y provocador de la sonrisa, la tarea resultaba más efectiva y mejor valorada por los clientes que cuando no había humor o cuando este era de un tipo no facilitador por ser compresible pero fuera de lugar o ser irrelevante (Davidson y Brown, 1989). 
Por tanto, hay ya algunos indicios de que el humor puede ser utilizado para mejorar la calidad de vida de las personas con discapacidad intelectual, pero se necesita aumentar considerablemente el total de conocimientos que actualmente se tienen sobre el tema. Saber, por ejemplo, cómo estas personas pueden ser entrenadas para apreciar mejor lo humorístico y cuales serían los diseños de aprendizaje más apropiados; o cómo se puede, en qué situaciones y de qué manera debe ser utilizado el sentido del humor por los profesionales para mejorar el trato y la efectividad de los apoyos, pueden ser algunos objetivos interesantes para la actividad investigadora sobre el tema.

En este sentido parece apropiado que las líneas de investigación que se tomen deban estar basadas mayormente en los pilares de un paradigma integrador, de carácter holístico, comprensivo, ecológico y contextual; pues en su fundamento teórico se aúnan y complemen$\tan$ aquellos enfoques que han venido resultando prácticos, pero insuficientes, en la investigación realizada por o para las personas que tienen necesidades educativas especiales (Rodríguez, 2005).

Las emociones positivas tienen efectos muy beneficiosos para el bienestar de todas las personas (Avia y Vázquez, 1998). Y son especialmente importantes para las personas con discapacidad intelectual pues pueden verse ayudadas a hacer frente de manera decisiva a las barreras que las gentes y el entorno les ponen por delante. El humor, la alegría, la diversión y otras emociones positivas generan una perspectiva personal positiva de la vida, y esto es importante para que los individuos puedan pensar con creatividad en los procesos de resolución de sus problemas vitales y tratar las dificultades diarias desde una posición de mayor fortaleza (Brown, 2007). Es necesario que todas estas ventajas sean previstas, también, para las personas con discapacidad intelectual en los planes y programas de apoyo que se diseñen para ellos pues, sin duda, que con su práctica aumentará su bienestar emocional y su calidad de vida.

\section{Agradecimientos}

El autor quisiera dar las gracias por su colaboración para la elaboración de este artículo al Profesor Dr. Ivan W. Brown, Centre of Excellence for Child Welfare, Faculty of Social Work, University of Toronto (Canadá) y al Profesor Dr. Pedro Ramiro, Facultad de Educación, Universidad de Cádiz. 


\section{Referencias}

Alemany, C. y Cabestrero, R. (2002). Humor, psicología y psicoterapia: estudios e investigaciones. En A. Rodríguez (Ed.). El valor terapéutico del humor. Bilbao: Desclée de Brouwer.

Amodia del la Riva, J. y Andrés, M. A. (2006). Trastorno de autismo y discapacidad intelectual. En AA.VV. Síndromes y apoyos. Panorámica desde la ciencia y las asociaciones. Madrid: FEAPS.

Arthur, A. R. (2003). The emotional lives of people with learning disability. British Journal of Learning Disabilities, 31 (1), 25-30.

Avia, M. D. y Vázquez, C. (1998). Optimismo inteligente. Madrid: Alianza.

Bernet, W. (2004). La importancia del humor en la psicoterapia con nińos y adolescentes. En W. F. Fry y W. A. Salameh (Ed). El humor y el bienestar en las intervenciones clínicas. Bilbao: Desclée de Brouwer.

Berry, P., Parson, G., Hyde, M. y Hilsdon, R. (1981). Observations of laughing and smiling in a group of moderately intellectually handicapped students. Exceptional Child, 28 (2), 128-132.

Brizzio, A., Carreras, A. y Casullo, M. M. (2006). Sentido del humor y sintomatología psicopatológica: un estudio con adolescentes y adultos jóvenes argentinos. Investigaciones Psicológicas, 11 (1).

Brown, I. W. (1994). Perception of humor in cartoon riddles by adults with intellectual disability . Perceptual and Motor Skills, 78 (3), 817-818.

Brown, I. W. (2007). El humor en las personas adultas con discapacidad intelectual. Comunicación personal por correo electrónico (22/3/2007).

Bruno, R. M., Johnson, J. M. y Simon, J. (1988). Perception of humor by learning disabled, mildly retarded, and nondisabled. Learning Disabilities Focus, 3 (2), 114-123.

Buxman, K. (2004). El humor en el tratamiento de los enfermos mentales. En W. F. Fry y W. A. Salameh (Ed). El humor y el bienestar en las intervenciones clínicas. Bilbao: Desclée de Brouwer.

Carbelo, B. (2005). El humor en la relación con el paciente. Barcelona: Masson.

Carbelo, B. (2006). Emociones positivas: humor positivo. Papeles del Psicólogo, 27 (1), 1830.

Carretero, H., Pérez, C. y Buela, G. (2006). Dimensiones de la apreciación del humor. Psicothema, 18 (3), 465--470.

Charlot, L. (2005). Use of behavioral equivalents for symptoms of mood disorders. En P. 
Sturneid (Ed.). Mood disorders in people with mental retardation. New York: National Association for Dually Diagnosed.

Davidson, I. F. W. K. y Brown, I. W. (1989). Using humour in counselling mentally retarded clients: a preliminary study. International Journal for the Advancement of Counselling, 12 (2), 93-104.

Dosen, A. (2005). Applying the developmental perspective in the psychiatric assesment and diagnosis of persons with intellectual disability: part I - assesment. Journal of Intellectual Disability Research, 49 (1), 1-8.

Fernández, J. D. (2002). Pedagogía del humor. En A. Rodríguez (Ed.). El valor terapéutico del humor. Bilbao: Desclée de Brouwer.

García, B. (2006). Programa para mejorar el sentido del humor. Madrid: Pirámide.

Glass, K. L. (2003). Comprehension of humor in children with nonverbal learning disabilities. University of Texas at Austin: Tesis doctoral no publicada.

Lyons, V. y Fitzgerald, M. (2004). Humor in autism and Asperger syndrome. Journal of Autism and Developmental Disorders, 34 (5), 521-531.

Martin, R. A. (2004). Sense of humor and physical health: theoretical issues, recent findings, and future directions. Humor. International Journal of Humor Research, 17 (1-2), 119.

McGhee, P. (2002). Understanding and promoting the development of children's humor. New Jersey: www.thelaughterremedy.com.

Nevo, O. (2004). El humor en la orientación profesional. En W. F. Fry y W. A. Salameh (Ed). El humor y el bienestar en las intervenciones clínicas. Bilbao: Desclée de Brouwer.

Paredes, D. (2005). Observaciones del humor en personas con discapacidad intelectual. Documento no publicado.

Pickering, E., Pickering, A. y Buchanan, M. (1987). LD and nonhandicapped boys' comprehension of cartoon humor. Learning Disability Quarterly, 10 (1), 45-51.

Polimeni, J. y Reiss, J. P. (2006). The first joke: exploring the evolutionary origins of humor. Evolutionary Psychology, 4 (1), 347-366.

Reddy, V., Williams, E. y Vaughan, A. (2002). Sharing humour and laughter in autism and Down's syndrome. British Journal of Psychology, 93 (2), 219-241.

Rieger, A. (2004). Make it just as normal as possible with humor. Mental Retardation, 42 (6), 427-444.

Rodríguez, A. (2002). La dimensión terapéutica del humor. En A. Rodríguez (Ed.). El valor 
terapéutico del humor. Bilbao: Desclée de Brouwer.

Rodríguez, A. (2005). Investigación sobre las necesidades educativas especiales: ¿qué y cómo investigar en Educación Especial? Electronic Journal of Research in Educational Psychology, 5-3 (1), 97-112. Obtenido el 1 de abril de 2007 en www.investigacionpsicopedagogica.org/revista/articulos/5/espannol/Art_5_41.pdf

Salameh, W. A. (2004). Un sistema de automejoramiento en competencias emocionales para el crecimiento personal y su más fácil absorción mediante el humor. En W. F. Fry y W. A. Salameh (Ed). El humor y el bienestar en las intervenciones clínicas. Bilbao: Desclée de Brouwer.

Seligman, M. P. (2003). La autentica felicidad. Barcelona: Vergara.

Short, E. J., Basili, L. A. y Schatschneider, C. W. (1993). Analysis of humor skills among elementary school students: comparisons of children with and without intellectual handicaps. American Journal on Mental Retardation, 98 (1), 63-73.

St. James, P. J. y Tager-Flusberg, H. (1994). A observational study of humor in autism and Down's syndrome. Journal of Autism and Developmental Disorders, 24 (5), 603-617.

Torradabella, P. y Corrales, J. (2002). Cómo desarrollar el sentido del humor. Las claves de la alegría interna. Barcelona: Océano. 